\title{
Stationary Non-Equilibrium States of Infinite Harmonic Systems
}

\author{
Herbert Spohn` and Joel L. Lebowitz ${ }^{\star \star}$ \\ Belfer Graduate School of Science, Yeshiva University, New York, NY 10033, USA
}

\begin{abstract}
We investigate the existence, properties and approach to stationary non-equilibrium states of infinite harmonic crystals. For classical systems these stationary states are, like the Gibbs states, Gaussian measures on the phase space of the infinite system (analogues results are true for quantum systems). Their ergodic properties are the same as those of the equilibrium states: e.g. for ordered periodic crystals they are Bernoulli. Unlike the equilibrium states however they are not "stable" towards perturbations in the potential.

We are particularly concerned here with states in which there is a nonvanishing steady heat flux passing through "every point" of the infinite system. Such "superheat-conducting" states are of course only possible in systems in which Fourier's law does not hold: the perfect harmonic crystal being an example of such a system. For a one dimensional system, we find such states (explicitely) as limits, when $t \rightarrow \infty$, of time evolved initial states $\mu_{i}$ in which the "left" and "right" parts of the infinite crystal are in "equilibrium" at different temperatures, $\beta_{L}^{-L} \neq \beta_{R}^{-1}$, and the "middle" part is in an arbitrary state. We also investigate the limit of these stationary $(t \rightarrow \infty)$ states as the coupling strength $\lambda$ between the "system" and the "reservoirs" goes to zero. In this limit we obtain a product state, where the reservoirs are in equilibrium at temperatures $\beta_{L}^{-1}$ and $\beta_{R}^{-1}$ and the system is in the unique stationary state of the reduced dynamics in the weak coupling limit.
\end{abstract}

\section{Introduction}

Our theoretical understanding of the properties of large, macroscopic size, objects is based to a great extent on the study of idealized model systems. Such models are particularly useful when it is possible to identify explicitely some observed behavior characteristic of macroscopic systems with properties of the models which appear,

* On leave of absence from the Fachbereich Physik der Universität München. Work supported by a Max Kade Foundation Fellowship

$\star \star \quad$ Research supported in part by NSF Grant MPS75-20638 
or take on essential new qualitative features, in the "thermodynamic" or infinite volume limit. Thus, for equilibrium systems, one can identify physical phase transitions with the appearance of singularities in the thermodynamic functions of various model systems in this limit. In many interesting cases, e.g. ferromagnetic spin systems, this phenomena can be studied in some detail.

For non-equilibrium systems the situation is much less satisfactory at the present time. We do not yet have any (dynamical) model systems in which even the simplest kinetic "laws", e.g. Fourier's law of heat conduction, can be shown to hold. Indeed the models for which non-equilibrium properties can be computed, e.g. the non-interacting gas and the perfect harmonic crystal corresponding to an ideal fluid and an ideal solid, do not obey any macroscopic kinetic laws [1]. We feel however that in spite of this the non-equilibrium properties of these ideal systems are worth investigating for what they can teach us about essential new features of large systems out of equilibrium. This is important since our present knowledge of nonequilibrium phenomena is so limited that we do not even know what features of the interactions are responsible for real systems obeying kinetic laws. We also do not know at present how to formulate, in a precise mathematical way, the statistical mechanics of stationary, current carrying, states of real systems although this is one of the simplest non-equilibrium phenomena. The present note is devoted to the investigation of such stationary states in harmonic systems.

Our work is related to earlier investigations by Lebowitz et al. [2-4] and other authors $[5,6]$ of the time evolution and stationary states of a finite harmonic crystal, e.g. a one dimensional chain of $N$-particles connected by harmonic springs, whose left and right ends are in contact with stochastic heat reservoirs at temperatures $\beta_{L}^{-1}$ and $\beta_{R}^{-1}$. When the temperature of the two reservoirs are equal, $\beta_{L}=\beta_{R}=\beta$, then the ensemble (probability) density of the system in its phase space, $\varrho\left(q_{1}, p_{1}, \ldots, q_{N}, p_{N} ; t\right)$ approaches, as $t \rightarrow \infty$, the canonical distribution $Z^{-1}$ $\exp \left[-\beta H\left(q_{1}, \ldots, p_{N}\right)\right]$. Here $q_{i}, p_{i}$ are the displacement and momentum of the $i$-th particle and the Hamiltonian has the form, $H=\frac{1}{2} \sum p_{i}^{2} / m_{i}+\frac{1}{2} \sum A_{i j} q_{i} q_{j}$. When $\beta_{L} \neq \beta_{R}$ the system ensemble density still approaches a stationary state in which however there will now be a constant energy (heat) flux, $J_{N}$, through the system going from the hot to the cold reservoir. For the one dimensional chain, with nearest neighbor couplings, $J_{N} \sim\left\langle q_{j} p_{j+1}\right\rangle$ where the expectation is to be taken in the stationary state.

To obtain more information about these stationary non-equilibrium states, it is necessary to specify the couplings between system and reservoirs. It was found in [2] that when the effect of each reservoir on the particles with which it is in contact is described by an Ornstein-Uhlenbeck process then all the stationary states of the system are given by Gaussian distributions. For the one dimensional chain, with equal masses, it was even possible to obtain explicitely the covariance matrix of the general stationary Gaussian state. It was then found that, for large $N$, the heat conductivity $\varkappa(N)$, defined as the heat flux $J_{N}$ divided by the "temperature gradient" $\left(\beta_{L}^{-1}-\beta_{R}^{-1}\right) / N$ grows like $N$ while the "kinetic temperature" (average kinetic energy) is constant throughout the chain (except very near the ends). This means in particular that Fourier's law is not obeyed: the heat flux is proportional to the temperature difference $\left(\beta_{L}^{-1}-\beta_{R}^{-1}\right)$ and not to the gradient; $J_{N} \rightarrow J \neq 0$ as $N \rightarrow \infty$. This property of the stationary state appears to hold for a wide range of harmonic 
systems and reservoir couplings [3]; at least whenever the spectrum of the force matrix $A$ has, for the infinite system, an absolutely continuous part which will generally be the case, when the system is perfectly ordered. The situation is quite different however for the isotopically disordered chain where the masses $m_{j}$ vary from site to site in a "random" way. In this case $J_{N} \rightarrow 0$ for almost all mass configurations $[7,4]$. Note however that $J_{N} \rightarrow 0$ still leaves open the question of whether Fourier's law is obeyed in the random system, i.e. does $x(N) \rightarrow \chi, 0<x<\infty$, in this case? Very recently this question was answered in the negative, at least for one dimension, by Papanicolaou [8] who showed that $x_{N} \sim N^{1 / 2}$ for the random chain. What happens in higher dimensions is still an open and very interesting problem. (It should be noted that the kinetic properties of an harmonic crystal may actually be relevant to the behavior of some real solids at very low temperatures when anharmonic effects are "negligible" [1].)

Papanicolaou's result was actually not derived for the chain with stochastic reservoirs rather it was for a model investigated by Rubin and Greer [9] and also by other workers $[10,11]$. In this model the reservoirs themselves consist of semiinfinite harmonic chains - the left "reservoir" consists of particles with index $j \in(-\infty, M-1]$ and the right "reservoir" of particles with index $j \in[N+1, \infty)$. At $t=0$ these reservoirs are assumed to be in thermal equilibrium with reciprocal temperatures $\beta_{L}$ and $\beta_{R}$. Rubin and Greer [9] then derive an expression for the heat flux through the system as $t \rightarrow \infty$ : cf. also Hemmer [11], O'Connor and Lebowitz [4] and others [10]. These authors do not however consider the full stationary probability distribution of the system much less that of the reservoirs. These reservoirs can actually be viewed as forming, together with the system, an infinite harmonic chain -with a particular initial measure. It is precisely this point of view which we adapt here and thereby place this investigation in the general context of finding the non-equilibrium behavior of large, formally infinite, systems.

The time evolution of infinite harmonic systems and the ergodic properties of their equilibrium states have been studied recently by Lanford and Lebowitz [12], Titulaer [13], and van Hemmen [14]. The existence of a time evolution $T_{t}^{*}$ was proven in [12] under very general conditions on the dynamical force matrix $A$. It was also shown there that the limit of finite volume canonical ensembles, at reciprocal temperatures $\beta^{-1}$, is a stationary Gaussian measure, $\mu_{\beta}$, on the phase space $\Omega^{\prime}$ of the infinite system : $\mu_{\beta}$ thus describes the equilibrium state of an infinite harmonic crystal. The ergodic properties of the dynamical system $\left(\Omega^{\prime}, T_{t}^{*}, \mu_{\beta}\right)$ were then shown to be directly related to the spectral properties of the matrix $A$. In particular, absolute continuity of the spectrum of $A$ is a necessary and sufficient condition for the dynamical system to be Bernoulli. This condition is generally satisfied for periodic (no disorder) harmonic crystals. If, on the other hand, the spectrum of $A$ contains some isolated eigenvalues, as would occur when there is a light impurity in an otherwise perfect crystal, then the system is not even ergodic. (It turns out that the time evolution $T_{t}^{*}$ in the phase space $\Omega^{\prime}$ is the dual of a flow $T_{t}$ in a space $\Omega$ [14]. It is the latter which will frequently be used, hence our notation.)

The implication of good ergodic properties, i.e. mixing which is itself implied by Bernoulliness, for an infinite system is that if such a system is locally disturbed away from equilibrium it will return to its equilibrium state. This return to equilibrium in the harmonic system is however not caused by any local collision mechanism, quite 
the opposite, it is due, as in the infinite ideal gas system, to local disturbances "flying off to infinity" unhindered, never to be seen again $[15,16]$. In the infinite ideal gas this escape is a direct and immediate consequence of the independent, straight line, motion of each particle. In the absence of particles with arbitrary small velocities there would be, in the infinite ideal gas no correlations between successive events, in a bounded region $\Lambda$, separated by a time interval greater than some fixed $t_{0}$. For the infinite harmonic crystal the independently moving objects which carry away the local information are not the particles but the normal modes or more precisely the running waves [1]. The fact, however, that these waves are not local objects makes the mathematical (and also the physical) analysis of the non-equilibrium behavior of the harmonic system more difficult and more interesting than that of the ideal gas.

The difference between the ideal gas and harmonic system becomes even more pronounced when we consider the time evolution of states which are "globally far" from any equilibrium state or for that matter from any stationary state. For the ideal gas any initial state with good clustering properties which has a certain amount of "uniformity" will eventually evolve into a state in which there are no correlations between the particles [17]. Conversely any spacially independent velocity distribution function determines, by a Poisson construction, a stationary state of the infinite system $[18,19]$. There are no such simple prescriptions for infinite harmonic systems. We prove an approach to stationarity under the condition that initially "far outside" the system is in equilibrium.

It is conceivable that a more general class of initial states approach a stationary state as $t \rightarrow \infty$.

The outline of this paper is as follows: In section two we describe our model system and the action of the time evolution operator $T_{t}^{*}$. This leads to a characterization of stationary Gaussian states - they are not specified by a finite number of parameters. In section three we prove the approach to a unique stationary Gaussian state of a one dimensional infinite harmonic system whose dynamical matrix $A$ has an absolutely continuous spectrum bounded away from zero, and whose initial state is one in which the "far left" side and "far right" side are each in "equilibrium" with temperatures $\beta_{L}^{-1}$ and $\beta_{R}^{-1}$. In section four we discuss the ergodic and stability properties of Gaussian stationary states. In section five we introduce a variable coupling $\lambda$ between the "system" and the "reservoir". We first investigate the weak coupling limit, $\lambda \rightarrow 0, t \rightarrow \infty, \lambda^{2} t=\tau$ fixed, of the (reduced) dynamics of the system. We then study the limit, as $\lambda \rightarrow 0$, of the stationary states $\mu_{\lambda}$, obtained as $t \rightarrow \infty$ from initial states considered in Section 3. We obtain a state $\mu_{0}$, where the reservoirs and the system are independent: the reservoirs are in equilibrium at temperature $\beta_{L}^{-1}$ and $\beta_{R}^{-1}$ and the state of the system is invariant under the reduced dynamics in the weak coupling limit. Finally, in section six, we apply our results to particular simple reservoirs: they consist of unit masses with nearest neighbor couplings of unit strength.

\section{Time Evolution and Stationary Gaussian States}

A general crystal lattice in $v$-dimensional space $R^{v}$ is specified by the group $\Gamma$ of translations carrying the lattice onto itself. $\Gamma$ is a discrete subgroup of the additive 
group $R^{v}$. As a group it is isomorphic to $Z^{v}$. For simplicity of notation, we assume that there is exactly one particle per unit cell (Bravais lattice). The points of our lattice represent the equilibrium positions of the particles making up the harmonic crystal. Let $q_{j} \in R^{v}$ be the displacement of the $j$-th particle, $j \in \Gamma$, from its equilibrium position and let $p_{j}$ be its conjugate momentum variable. In the harmonic approximation the equations of motion $\operatorname{read}[1,20]$ :

$$
d q_{j} / d t=p_{j}, \quad d p_{j} / d t=-\sum_{i} A_{j i} q_{i},
$$

where we have made the canonical transformation $q_{j} \rightarrow m_{j}^{-1 / 2} q_{j}, p_{j} \rightarrow m_{j}^{1 / 2} p_{j} ; m_{j}$ the mass of the $j$-th particle. $A$ is called the interaction or force matrix $\left[A_{i j}=\left(m_{i} m_{j}\right)^{-1} V_{i j} ; \frac{1}{2} \sum V_{i j} q_{i} q_{j}\right.$ is the potential energy $]$.

To formulate precisely the dynamics of the infinite system, heuristically given by (2.1), we need various spaces of sequences $\left(\xi_{j}\right)_{j \in \Gamma}$ taking (real) values in $R^{v}$. Let $d(\Gamma)$ be the space of finite sequences (i.e. $\xi_{j}=0$ for all but finitely many $j$ ). Following [14] we introduce the family $\left\{\|\cdot\|_{m} \mid m \in N\right\}$ of norms on $d(\Gamma)$

$$
\|\xi\|_{m}^{2}=\sum_{j \in \Gamma}\left|\xi_{j}\right|^{2}\left(1+j^{2}\right)^{m}
$$

The completion of $d(\Gamma)$ with respect to the norm $\|\cdot\|_{m}$ is the Hilbert space $s_{m}$. $s_{0}$ will also be denoted by $l^{2}$. $s_{-m}$ and $s_{m}$ are dual to each other under the mapping $\langle\xi \mid x\rangle$ $=\sum_{j \in \Gamma} \xi_{j} x_{j}$. The space $s$ of rapidly decreasing sequences is $s=\bigcap_{m=0}^{\infty} s_{m}$ and its dual, the space of polynomially bounded sequences, is $s^{\prime}=\bigcup_{m=0}^{\infty} s_{-m} . s$ equipped with the collection of norms $\left\{\|\cdot\|_{m} \mid m \in N\right\}$ is a nuclear space [14]. We equip $s^{\prime}(\Gamma)$ with its weak* topology and the $\sigma$-algebra inherited from that topology. Then a convenient phase space for the infinite harmonic system is the measurable space $\Omega^{\prime}$ $=s^{\prime}(\Gamma) \oplus s^{\prime}(\Gamma)$.

For the interaction matrix $A$ we now assume:

(i) $A$ is a bounded operator on each $s_{-m}, m \geqq 0$.

Rewriting the equation of motion as

$$
\frac{d}{d t}\left(\begin{array}{l}
p \\
q
\end{array}\right)=\left(\begin{array}{rr}
0 & -A \\
I & 0
\end{array}\right)\left(\begin{array}{l}
p \\
q
\end{array}\right)
$$

we see $[12,14]$ that, by exponentiating, the solutions of $(2.3)$ define a flow $T_{t}^{*}$ on $s_{-m} \oplus s_{-m}$ for each $m \geqq 0$ and therefore also on the phase space $\Omega^{\prime}$. By duality, $A^{*}$ is bounded on $s_{m}, m \geqq 0$. Thus, the solutions of the "dual equations of motion"

$$
\frac{d}{d t}\left(\begin{array}{l}
\xi \\
\eta
\end{array}\right)=\left(\begin{array}{cc}
0 & I \\
-A^{*} & 0
\end{array}\right)\left(\begin{array}{l}
\xi \\
\eta
\end{array}\right)
$$

define a flow $T_{t}$ on each $s_{m} \oplus s_{m}$ and therefore also on $s(\Gamma) \oplus s(\Gamma) \equiv \Omega$. Obviously, $T_{t}^{*}$ is the dual group of transformations of $T_{t}$.

Condition (i) has a direct physical interpretation. Since $s_{-m}$ consists of polynomially growing sequences, $A$ will be bounded if $A_{i j}$ decreases sufficiently fast 
for $|i-j| \rightarrow \infty$, i.e. if the forces drop off rapidly between particles whose equilibrium positions are far away. A sufficient condition for (i) to hold [12] is

$$
\sup _{i \in \Gamma} \sum_{j \in \Gamma}\left|A_{i j}\right|\left[1+(i-j)^{2}\right]^{m}<\infty
$$

for each $m \geqq 0$.

In order for our harmonic system to be a model of a crystal it is essential that the equilibrium positions, $q_{i}=0$, correspond to at least a local minimum in the potential energy. This means that $A$ should be positive (and therefore symmetric). We shall therefore assume:

(ii) $A$ is a strictly positive (bounded) operator on $l^{2}(\Gamma)$, i.e. $\langle\xi \mid A \xi\rangle \geqq 0$ for $\xi \in l^{2}(\Gamma)$ with equality holding only if $\xi=0$. Later we shall impose further conditions on the behavior of $A$ near zero.

We now look for Gaussian measures invariant under the time evolution $T_{t}^{*}$. Since the phase space $\Omega^{\prime}$ is the dual of a nuclear space $\Omega$, any probability measure $\mu$ on $\Omega^{\prime}$ is by Minlos' theorem $[21,14]$ uniquely defined by its Fourier transform $F \mu$ which is a positive definite continuous function on $\Omega$ with $F \mu(0)=1$. For a meanzero non-degenerate Gaussian measure the Fourier transform is $\exp \left[-\frac{1}{2}\langle\xi \mid Q \xi\rangle\right]$, where $\langle\xi \mid Q \xi\rangle$ is a bilinear, continuous, strictly positive form on $\Omega$. $Q$ is called the covariance matrix. For such a Gaussian measure to be stationary it is clearly both necessary and sufficient that

$$
T_{t}^{*} Q T_{t}=Q
$$

for all $t \in R$. Differentiating and using (2.3) and (2.4), we obtain that a necessary and sufficient condition for stationarity is that $Q$ have the form

$$
Q=\left(\begin{array}{cc}
A Q_{1} & Q_{2} \\
-Q_{2} & Q_{1}
\end{array}\right)
$$

with $Q_{1}$ strictly positive (possibly unbounded) on $l^{2}(\Gamma), Q_{2}$ anti self-adjoint on $l^{2}(\Gamma)$, i.e. $Q_{2}^{*}=-Q_{2}$, and $\left[Q_{1}, A\right]=0=\left[Q_{2}, A\right]$. The choice $Q_{1}=\beta^{-1} A^{-1}, Q_{2}=0$, yields the covariance matrix of the equilibrium state at temperature $\beta^{-1}$. [Here, we have to assume that $\left\langle\xi \mid A^{-1} \xi\right\rangle$ is continuous on $s(\Gamma)$.]

Remarks. (i) While our discussion has been couched in language appropriate to the infinite system condition (2.6) also holds for finite systems; $A$ finite system in a region $\Lambda$ corresponds to setting $q_{i} \equiv p_{i} \equiv 0$ for $i \notin \Lambda \subset \Gamma$ and $A_{i j}=0$ unless $i, j \in \Lambda$, [12].

(ii) We also note that due to the linearity of the equations of motion (2.1), every set of "homogeneous" expectation values $\left\{\left\langle q_{i}^{n_{1}} q_{j}^{n_{2}} \ldots p_{l}^{n_{l}}\right\rangle\right\}, n_{\alpha}$ non-negative integers and $\sum n_{\alpha}=n$ fixed, obeys an autonomous equation of motion. Hence the covariance matrix of any stationary state of the finite or infinite harmonic system (not only Gaussian states) must have the form (2.6). Conversely, given a covariance matrix $Q$ of the form (2.6) we can always construct at least one stationary state, the Gaussian one, with this covariance. For the finite system there will of course be many (an infinite number) stationary states with the same $Q$ but we do not know whether the same is true for the infinite system. 
To get some insight into the nature of the stationary states defined by (2.6), let us assume that the spectrum of $A$ is non-degenerate. Then $Q_{1}=g(A), Q_{2}=f(A)$. If the system is finite, then, for $Q_{2}$ to be real, $f$ has to be a real function. By the anti selfadjointness this implies $Q_{2}=0$ so that we always have $\left\langle q_{j} \mid Q p_{k}\right\rangle=0$ for finite systems. This implies in particular (the obvious fact) that there can be no steady heat flow through an isolated finite system. Indeed for a finite system the invariant Gaussian measures are simply Gaussians formed by the normal modes $[1,20]$ with different weights. However, if the system is infinite, then $f$ does not have to be a real function and therefore $Q_{2}$ does not have to be zero. This can be seen explicitely for the one dimensional system with only nearest neighbor interaction where $\Gamma=Z$ and $A$ is a tridiagonal matrix with $A_{i i}=2, A_{i j}=-1$ if $|i-j|=1, A_{i j}=0$ otherwise. If we now choose $\left(Q_{2}\right)_{i j}=j-i$ if $|i-j|=1,\left(Q_{2}\right)_{i j}=0$ otherwise, then $Q_{2}=i\left(A-\frac{1}{4} A^{2}\right)^{1 / 2}$. Thus, an infinite harmonic system will have many stationary non-equilibrium Gaussian measures. In the next section we show that some of these states can be obtained as the limit, when $t \rightarrow \infty$, of physically interesting initial conditions.

\section{Approach to Stationary State}

We consider an infinite harmonic chain, $\Gamma=Z$, and let $P_{L}, P, P_{R}$ be the projection on $(-\infty, M-1],[M, N],[N+1, \infty),-\infty<M+1 \leqq N<\infty$, respectively. We shall sometimes refer to the segment $(-\infty, M-1]$ as the "left reservoir", to the segment $[M, N]$ as the "system" and to the segment $[N+1, \infty)$ as the "right reservoir" even though they are all part of one in finite system. Initially, the system is in an arbitrary state $\varrho$ and the reservoirs are in "equilibrium" with temperatures $\beta_{L}^{-1}$ and $\beta_{R}^{-1}$, i.e. their states are the equilibrium states $\mu_{\beta_{L}}$ and $\mu_{\beta_{R}}$ of the semi-infinite chains with interaction matrices $P_{L} A P_{L}$ and $P_{R} A P_{R}$. In order for these states to be well defined, we have to assume that $\left\langle\xi \mid\left(P_{L} A P_{L}\right)^{-1} \xi\right\rangle$ and $\left\langle\xi \mid\left(P_{R} A P_{R}\right)^{-1} \xi\right\rangle$ are continuous on $P_{L} S(Z)$ and $P_{R} S(Z)$, respectively. Thus at $t=0$ the state of the infinite chain is,

$$
\mu_{i}=\mu_{\beta_{L}} \otimes \varrho \otimes \mu_{\beta_{R}}
$$

and we are interested in

$$
\lim _{t \rightarrow \infty} \mu_{i} \circ T_{t}^{*}
$$

The limit in (3.2) is to be understood in the weak sense.

As we shall see later, whenever the limit (3.2) exists it will define a Gaussian measure on the phase space $\Omega^{\prime}$. To see why this is so we can think of the time evolution as composed of noninteracting waves (normal modes) propagating through the infinite crystal. As $t \rightarrow \infty$, all initial local information streams off and we merely see a "superposition" of waves' travelling to the right with "weights" appropriate to $\mu_{\beta_{L}}$ and waves travelling to the left with weights appropriate to $\mu_{\beta_{R}}$. If $\beta_{L}<\beta_{R}$, then in the final steady state more "waves" will travel to the right than to the left producing a steady energy flow through the chain.

Given this interpretation of the steady state we expect, and will later show, that the limit (3.2) is independent of $\varrho$ and of the (finite) interval $[M, N]$. A multiplication of $\mu_{i}$ with a density $f$ which corresponds to a "local" change in the initial state $\mu_{i}$ will 
also not alter the final state. However, the final state will have a very sensitive dependence on the dynamics, e.g. if there is a heavy impurity at the origin with mass $m$, then as $m$ increases the heat flow throughout the whole system will decrease.

There will be no such effect, of course, when $\beta_{L}=\beta_{R}$ and the final state is one of equilibrium. It is indeed their stability to local perturbations which distinguishes the equilibrium states from the other stationary states (cf. next section).

The theorems of this section are not pushed to utmost generality. The method certainly applies, whenever the region $\Lambda$ of the system blown up by the range of the interaction is a finite subset of $\Gamma$ and the state of the reservoir in $\Gamma \backslash \Lambda$ is a Gaussian measure invariant under the "decoupled" time evolution $T_{t}^{0 *}$.

The existence of the limit (3.2) is related to a scattering problem. This is a well defined problem in itself and we study it in the next few paragraphs. One compares the true time evolution $T_{t}$ with the "unperturbed" time evolution $T_{t}^{0}$ which is generated by (2.4) with $A$ replaced by $A_{0}=P_{L} A P_{L}+P A P+P_{R} A P_{R}$. $T_{t}^{0}$ is the time evolution when the system and reservoirs are isolated from each other. We define the wave operator $\Delta$ as

$$
s-\lim _{t \rightarrow \infty} T_{-t}^{0} T_{t}=\Delta,
$$

whenever this limit exists, where $s$-lim denotes the strong limit in $l^{2}(Z) \oplus l^{2}(Z)$. The existence of the wave operator means that waves (excitations) far away from the system propagate practically (i.e. in the $l^{2}$-norm) according to the unperturbed time evolution. The scattering problem considered here is somewhat different from the one usually considered in quantum or classical mechanics. $T_{t}$ and $T_{t}^{0}$ are not unitary groups in $l^{2}(Z) \oplus l^{2}(Z)$. Furthermore, depending on the behavior of $A$ near zero, $\Delta$ can be unbounded and (3.3) has to be understood in a generalized sense. On the other hand, the difference of the generators of $T_{t}$ and $T_{t}^{0}$ is just $A-A_{0}=A_{c}=\left(P_{L}\right.$ $\left.+P_{R}\right) A P+P A\left(P_{L}+P_{R}\right)+P_{L} A P_{R}+P_{R} A P_{L}$. If the interaction matrix $A$ comes from a finite range interaction, we have a perturbation of finite rank. Therefore, one expects the existence of the wave operator under fairly general conditions.

Theorem 1. Let the interaction matrices $A$ and $A_{0}$ generate the flows $T_{t}$ and $T_{t}^{0}$ as in (2.4). If the spectrum of $A$, in $l^{2}(\Gamma)$, is absolutely continuous, if $A$ and $A_{0}$ are bounded away from zero and if $A-A_{0}$ is of trace class, then the wave operator

$$
S-\lim _{t \rightarrow \infty} T_{-t}^{0} T_{t}=\Delta
$$

exists in $l^{2}(\Gamma) \oplus l^{2}(\Gamma)$.

Proof. By [22, X, Theorem 4.4]

$$
s-\lim _{t \rightarrow \infty} e^{-i A_{0} t} e^{i A t}=\tilde{\Delta}
$$

exists in $l_{\mathbb{C}}^{2}(\Gamma)$, the complexification of $l^{2}(\Gamma)$. We shall now show that (3.5) implies (3.4). Writing out (3.4) more explicitely, we find that it corresponds to

$$
\begin{aligned}
& \underset{t \rightarrow \infty}{s-\lim _{t \rightarrow \infty}}\left\{\left[\begin{array}{cc}
\cos \left(A_{0}^{1 / 2} t\right) & -A_{0}^{-1 / 2} \sin \left(A_{0}^{1 / 2} t\right) \\
A_{0}^{1 / 2} \sin \left(A_{0}^{1 / 2} t\right) & \cos \left(A_{0}^{1 / 2} t\right)
\end{array}\right]\left[\begin{array}{cc}
\cos \left(A^{1 / 2} t\right) & A^{-1 / 2} \sin \left(A^{1 / 2} t\right) \\
-A^{1 / 2} \sin \left(A^{1 / 2} t\right) & \cos \left(A^{1 / 2} t\right)
\end{array}\right]-\Delta\right\} \\
& =0 \text {. }
\end{aligned}
$$


In successive steps, we will convert (3.5) into (3.6). that

Since $A$ is bounded away from zero, then by [22, X, Theorem 4.7$]$ we also have

$$
s-\lim _{t \rightarrow \infty} e^{-i A_{0}^{1 / 2} t} e^{i A^{1 / 2} t}=\tilde{\Delta}
$$

in $l_{\mathbb{C}}^{2}(\Gamma)$. Since $A$ and $A_{0}$ are real, (3.7) implies

$$
\begin{aligned}
& s-\lim _{t \rightarrow \infty}\left\{\cos \left(A_{0}^{1 / 2} t\right) \cos \left(A^{1 / 2} t\right)+\sin \left(A_{0}^{1 / 2} t\right) \sin \left(A^{1 / 2} t\right)\right\}=\Delta_{1} \\
& { }_{s-\lim _{t \rightarrow \infty}}\left\{\cos \left(A_{0}^{1 / 2} t\right) \sin \left(A^{1 / 2} t\right)-\sin \left(A_{0}^{1 / 2} t\right) \cos \left(A^{1 / 2} t\right)\right\}=\Delta_{2}
\end{aligned}
$$

in $l^{2}(\Gamma)$. We note the intertwining property $\Delta_{1} A^{1 / 2}=A_{0}^{1 / 2} \Delta_{1}, \Delta_{2} A^{1 / 2}=A_{0}^{1 / 2} \Delta_{2}$. Let us define

$$
\begin{aligned}
\Delta & =\left(\begin{array}{cc}
1 & 0 \\
0 & A_{0}^{1 / 2}
\end{array}\right)\left(\begin{array}{cc}
\Delta_{1} & \Delta_{2} \\
-\Delta_{2} & \Delta_{1}
\end{array}\right)\left(\begin{array}{cc}
1 & 0 \\
0 & A^{-1 / 2}
\end{array}\right)=\left(\begin{array}{cc}
A_{0}^{-1 / 2} & 0 \\
0 & 1
\end{array}\right)\left(\begin{array}{cc}
\Delta_{1} & \Delta_{2} \\
-\Delta_{2} & \Delta_{1}
\end{array}\right)\left(\begin{array}{cc}
A^{1 / 2} & 0 \\
0 & 1
\end{array}\right) \\
& =\left(\begin{array}{cc}
\Delta_{1} & \Delta_{2} A^{-1 / 2} \\
-\Delta_{2} A^{1 / 2} & \Delta_{1}
\end{array}\right) .
\end{aligned}
$$

(3.8) can be rewritten as

$$
\begin{aligned}
& \operatorname{s-lim}_{t \rightarrow \infty}\left\{\left(\begin{array}{cc}
\cos \left(A^{1 / 2} t\right) & \sin \left(A^{1 / 2} t\right) \\
-\sin \left(A^{1 / 2} t\right) & \cos \left(A^{1 / 2} t\right)
\end{array}\right)\right. \\
& \left.-\left(\begin{array}{cc}
\cos \left(A_{0}^{1 / 2} t\right) & \sin \left(A_{0}^{1 / 2} t\right) \\
-\sin \left(A_{0}^{1 / 2} t\right) & \cos \left(A_{0}^{1 / 2} t\right)
\end{array}\right)\left(\begin{array}{cc}
\Delta_{1} & \Delta_{2} \\
-\Delta_{2} & \Delta_{1}
\end{array}\right)\right\}=0 .
\end{aligned}
$$

Multiplying with matrices as in (3.9), we obtain

$$
\begin{aligned}
& s-\lim _{t \rightarrow \infty}\left\{\left(\begin{array}{cc}
\cos \left(A^{1 / 2} t\right) & A^{-1 / 2} \sin \left(A^{1 / 2} t\right) \\
-\sin \left(A^{1 / 2} t\right) & A^{-1 / 2} \cos \left(A^{1 / 2} t\right)
\end{array}\right)\right. \\
& \left.-\left(\begin{array}{cc}
\cos \left(A_{0}^{1 / 2} t\right) & A_{0}^{-1 / 2} \sin \left(A_{0}^{1 / 2} t\right. \\
-\sin \left(A_{0}^{1 / 2} t\right) & A_{0}^{-1 / 2} \cos \left(A_{0}^{1 / 2} t\right.
\end{array}\right) \Delta\right\}=0 \\
& { }_{t \rightarrow \infty}^{s-\lim _{t \rightarrow \infty}}\left\{\left(\begin{array}{cc}
A^{1 / 2} \cos \left(A^{1 / 2} t\right) & \sin \left(A^{1 / 2} t\right) \\
-A^{1 / 2} \sin \left(A^{1 / 2} t\right) & \cos \left(A^{1 / 2} t\right)
\end{array}\right)\right. \\
& \left.-\left(\begin{array}{cc}
A_{0}^{1 / 2} \cos \left(A_{0}^{1 / 2} t\right) & \sin \left(A_{0}^{1 / 2} t\right) \\
-A_{0}^{1 / 2} \sin \left(A_{0}^{1 / 2} t\right) & \cos \left(A_{0}^{1 / 2} t\right)
\end{array}\right) \Delta\right\}=0 .
\end{aligned}
$$

The first line in (3.11) and the second line in (3.12) are equivalent to $\|\left(T_{t}\right.$ $\left.-T_{t}^{0} \Delta\right) \xi \| \rightarrow 0$ as $t \rightarrow \infty$. Since $A_{0}$ is bounded away from zero $\left\|\left(T_{-t}^{0} T_{t}-\Delta\right) \xi\right\|$ $=\left\|T_{-t}^{0}\left(T_{t}-T_{t}^{0} \Delta\right) \xi\right\| \leqq a\left\|\left(T_{t}-T_{t}^{0} \Delta\right) \xi\right\|$, which proves assertion (3.4).

Let us now return to the existence of the limit (3.2). In the following, expressions such as $\left(P_{L} A P_{L}\right)^{-1}$ will always be considered as operators on $l^{2}(Z) .\left(P_{L} A P_{L}\right)^{-1}$ is defined by the spectral theorem on $P_{L} l^{2}(Z)$ and then extended by zero to the whole space. 
Theorem 2. Let the initial state of the infinite harmonic chain be $f \mu_{i}$ with $\mu_{i}$ defined as in (2.1) and $f \in L^{1}\left(\Omega^{\prime}, \mu_{i}\right)$. If the spectrum of the interaction matrix $A$ is absolutely continuous and bounded away from zero and if $A_{c}$ is of trace class for all finite intervals $[M, N]$, then

$$
\lim _{t \rightarrow \infty}\left[f \mu_{i}\right] \circ T_{t}^{*}=\mu
$$

exists in the weak sense. The final state $\mu$ is independent of $\varrho, f$, and $[M, N]$. $\mu$ is a Gaussian measure on $\Omega^{\prime}$ with covariance matrix

$$
\Delta^{*}\left(Q_{L}+Q_{R}\right) \Delta=\Delta^{*}\left(\begin{array}{cc}
\beta_{L}^{-1} P_{L}+\beta_{R}^{-1} P_{R} & 0 \\
0 & \beta_{L}^{-1}\left(P_{L} A P_{L}\right)^{-1}+\beta_{R}^{-1}\left(P_{R} A P_{R}\right)^{-1}
\end{array}\right) \Delta,
$$

where $\Delta$ is the wave operator of (3.3).

Proof. We show the convergence of the Fourier transforms. Let $f \in L^{1}\left(\Omega^{\prime}, \mu_{i}\right)$ depend only on finitely many coordinates in $\left[M^{\prime}, N^{\prime}\right] \supset[M, N]$ and let $\left(1-P_{0}\right)$ be the projection on $\left[M^{\prime}, N^{\prime}\right]$. Let $R_{1}=P_{0}\left(Q_{L}^{-1}+Q_{R}^{-1}\right) P_{0}, R_{2}=\left(1-P_{0}\right)\left(Q_{L}^{-1}+Q_{R}^{-1}\right) P_{0}$ and $R_{3}=\left(1-P_{0}\left(\left(Q_{L}^{-1}+Q_{R}^{-1}\right)\left(1-P_{0}\right)\right.\right.$. Since $\mu_{\beta_{L}}$ and $\mu_{\beta_{R}}$ are Gaussian measures, we obtain for all $\xi \in \Omega$

$$
\begin{aligned}
& {\left[f\left(\mu_{\beta_{L}} \otimes \varrho \otimes \mu_{\beta_{R}}\right)\right]\left(\exp \left(i\left\langle T_{t} \xi \mid \cdot\right\rangle\right)\right)} \\
& =\exp \left(-\frac{1}{2}\left\langle T_{t} \xi \mid R_{1}^{-1} T_{t} \xi\right\rangle\right) . \\
& c \int d x \exp \left[\left\langle x \mid\left(1-R_{2} R_{1}^{-1}\right) T_{t} \xi\right\rangle-\frac{1}{2}\left\langle x \mid\left(R_{3}-R_{2} R_{1}^{-1} R_{2}^{*}\right) x\right\rangle\right] f(x) \varrho(x),
\end{aligned}
$$

where $c$ is a normalization constant and the integration is over $R^{2\left(N^{\prime}-M^{\prime}+1\right)}$. Since $A$ has an absolutely continuous spectrum the Riemann-Lebesgue lemma implies

$$
\lim _{t \rightarrow \infty}\left\langle x \mid\left(1-R_{2} R_{1}^{-1}\right) T_{t} \xi\right\rangle=0
$$

for all $x \in R^{2\left(N^{\prime}-M^{\prime}+1\right)}$ and all $\xi \in \Omega$. By Lebesgue's dominated convergence, the second factor in (3.15) converges to one as $t \rightarrow \infty$. Let $T_{t}^{\prime}$ be generated by $P_{0}\left(P_{L} A P_{L}\right.$ $\left.+P_{R} A P_{R}\right) P_{0}+\left(1-P_{0}\right) A\left(1-P_{0}\right)$, an operator bounded away from zero. Then, by Theorem 1,

$$
\lim _{t \rightarrow \infty} T_{-t}^{\prime} T_{t}=\Delta^{\prime}
$$

exists. Furthermore, since $T_{t}^{0 *}\left(R_{1}\right)^{-1} T_{t}^{0}=\left(R_{1}\right)^{-1}$,

$$
\begin{aligned}
& \quad\left\langle T_{t} \xi \mid\left(R_{1}\right)^{-1} T_{t} \xi\right\rangle-\left\langle\Delta^{\prime} \xi \mid\left(R_{1}\right)^{-1} \Delta^{\prime} \xi\right\rangle \mid \\
& \quad \leqq\left\|\left(T_{-t}^{\prime} T_{t}-\Delta^{\prime}\right) \xi\right\|\left\|R_{1}^{-1} T_{-t}^{\prime} T_{t} \xi\right\|+\left\|R_{1}^{-1} \xi\right\|\left\|\left(T_{-t}^{\prime} T_{t}-\Delta^{\prime}\right) \xi\right\| .
\end{aligned}
$$

Therefore the first factor in (3.15) converges to $\left.\left.\exp \left(-\frac{1}{2}\langle\xi| \Delta^{\prime *}\left(R_{1}\right)^{-1}\right) \Delta^{\prime} \xi\right\rangle\right)$. If $M^{\prime}=M, N^{\prime}=N$, then $\left\langle\xi \mid \Delta^{\prime *} R_{1}^{-1} \Delta^{\prime} \xi\right\rangle=\left\langle\xi \mid \Delta^{*}\left(Q_{L}+Q_{R}\right) \Delta \xi\right\rangle$.

The continuity of this form is obvious and by $(3.20)\left\langle\xi \mid \Delta^{*}\left(Q_{L}+Q_{R}\right) \Delta \xi\right\rangle$ is strictly positive on $\Omega$. Therefore $\exp \left[-\frac{1}{2}\left\langle\xi \mid \Delta^{*}\left(Q_{L}+Q_{R}\right) \Delta \xi\right\rangle\right]$ is the Fourier transform of a Gaussian measure $\mu$ on $\Omega^{\prime}$. Altogether we have shown that $\lim _{t \rightarrow \infty} \mu_{i} \circ T_{t}^{*}$ 
$=\mu$. Obviously $\mu$ is independent $\varrho$. Let $\mathscr{A}$ be the set of all functions in $L^{1}\left(\Omega^{\prime}, \mu_{i}\right)$ depending on finitely many coordinates only. If $f=1$ and $M^{\prime}<M, N^{\prime}>N$, then, since the initial state is unchanged, $\left\langle\xi \mid \Delta^{\prime *} R_{1}^{-1} \Delta^{\prime} \xi\right\rangle=\left\langle\xi \mid \Delta^{*}\left(Q_{L}+Q_{R}\right) \Delta \xi\right\rangle$, which implies that $\mu$ is independent of any density $f \in \mathscr{A}$. Since $\mathscr{A}$ is norm-dense in $L^{1}\left(\Omega^{\prime}, \mu_{i}\right)$ there exists a sequence $f_{n} \in \mathscr{A}$ such that $\left\|f_{n}-f\right\| \rightarrow 0$, for any $f \in L^{1}\left(\Omega^{\prime}, \mu_{i}\right)$. Hence for all measurable and bounded functions $g: \Omega^{\prime} \rightarrow \mathbb{C}$

$$
\left|\left(f \mu_{i}\right)\left(g \circ T_{t}^{*}\right)-\mu(g)\right| \leqq\left|\left(f_{n} \mu_{i}\right)\left(g \circ T_{t}^{*}\right)-\mu(g)\right|+\sup |g|\left\|f_{n}-f\right\|_{1} .
$$

Therefore, $\lim _{t \rightarrow \infty}\left(f \mu_{i}\right) \circ T_{t}^{*}=\mu$ for all $f \in L^{1}\left(\Omega^{\prime}, \mu_{i}\right)$.

Finally, we have to show that $\mu$ is independent of $[M, N]$. Let $\left[M_{1}, N_{1}\right] \subset Z$ be some finite interval and $\mu_{i}^{1}$ be the corresponding initial state as in (3.1). By the same argument as above $\lim _{t \rightarrow \infty} \mu_{i}^{1} \circ T_{t}^{*}=\mu^{1}$ exists. In (3.15) let $M^{\prime}=\min \left(M, M_{1}\right)$, $N^{\prime}=\max \left(N, N_{1}\right)$ and $f=1$. Then the Fourier transform of both $\mu$ and $\mu^{1}$ is $\exp \left(-\frac{1}{2}\left\langle|\xi| \Delta^{\prime *}\left(R_{1}\right)^{-1} \Delta^{\prime} \xi\right\rangle\right)$ which proves $\mu^{1}=\mu$.

By (3.9) we find for the covariance matrix $Q$ of $\mu$

$$
Q=\left(\begin{array}{cc}
1 & 0 \\
0 & A^{-1 / 2}
\end{array}\right)\left(\begin{array}{cc}
Q_{1} & Q_{2} \\
-Q_{2} & Q_{1}
\end{array}\right)\left(\begin{array}{cc}
1 & 0 \\
0 & A^{-1 / 2}
\end{array}\right)
$$

with

$$
\begin{aligned}
& Q_{1}=\beta_{L}^{-1}\left(\Delta_{1}^{*} P_{L} \Delta_{1}+\Delta_{2}^{*} P_{L} \Delta_{2}\right)+\beta_{R}^{-1}\left(\Delta_{1}^{*} P_{R} \Delta_{1}+\Delta_{2}^{*} P_{R} \Delta_{2}\right) \\
& Q_{2}=\beta_{L}^{-1}\left(\Delta_{1}^{*} P_{L} \Delta_{2}-\Delta_{2}^{*} P_{L} \Delta_{1}\right)+\beta_{R}^{-1}\left(\Delta_{1}^{*} P_{R} \Delta_{2}-\Delta_{2}^{*} P_{R} \Delta_{1}\right) .
\end{aligned}
$$

If $\beta_{L}=\beta_{R}=\beta$, then, since $\tilde{\Delta} * \tilde{\Delta}=1$, we obtain

$$
Q=\beta^{-1}\left(\begin{array}{cc}
1 & 0 \\
0 & A^{-1}
\end{array}\right) \text {. }
$$

We recognize in the present context, the return to equilibrium as a consequence of the properties of the wave operator $\tilde{\Delta}$.

Using this result, we can rewrite $Q$ as

$$
Q=\beta_{R}^{-1}\left(\begin{array}{cc}
1 & 0 \\
0 & A^{-1}
\end{array}\right)+(\Delta T) Q^{\prime},
$$

where $\Delta T$ is the temperature difference between the "reservoirs". This shows that the mixed moments $\left\langle p_{j} \cdot q_{i}\right\rangle$ (in particular the heat flow) are proportional on the temperature difference between the (infinitely) "far left" and "far right" sides of the system at $t=0$.

Remark. $A$ bounded away from zero can always be achieved by introducing at every lattice point a (possible very weak) harmonic restoring force. At the expense of some formal complications, one could adopt less drastic conditions for the behavior of $A$ near zero $[12,14]$. 


\section{Ergodic and Stability Properties of Stationary Gaussian States}

The ergodic properties of the dynamical system $\left(\Omega^{\prime}, T_{t}^{*}, \mu\right)$, where $\mu$ is any stationary Gaussian measure, yield information about the time evolution of local deviations from the stationary state $\mu$. Since harmonic systems have no local mechanism of dissipation, small disturbances of any stationary state should simply fly off to infinity. Therefore, we expect strong ergodic properties. In fact, we show that the ergodic properties of $\left(\Omega^{\prime}, T_{t}^{*}, \mu\right)$ are independent of the measure $\mu$ and depend only on the spectrum of the interaction matrix $A$. In particular, if the spectrum of $A$ is absolutely continuous then these dynamical systems are Bernoulli and their Kolmogorov-Sinai entropy is infinite. Thus the dynamical systems $\left(\Omega^{\prime}, T_{t}^{*}, \mu\right)$ are isomorphic to each other in the sense of ergodic theory [23].

Theorem 3. Let $\mu$ be a $T_{t}^{*}$-invariant Gaussian measure on $\Omega^{\prime}$. Then the dynamical system $\left(\Omega^{\prime}, T_{t}^{*}, \mu\right)$ is

a) ergodic if and only if $A$ acting on $l_{\mathbb{C}}^{2}(\Gamma)$, has no point spectrum,

b) a Bernoulli flow if and only if $A$ has absolutely continuous spectrum.

Proof. Propositions 4.2 and 4.3 of [12] still hold, since one uses there only the fact that $\mu$ is a $T_{t}^{*}$-invariant Gaussian measure. Let $h_{1}$ be the (real) closed subspace of Gaussian random variables in $L^{2}(\mu)$ and $U_{1}(t)$ be the orthogonal group induced by $T_{t}^{*}$. We have to relate the spectral properties of $U_{1}(t)$ on $\left(h_{1}\right)_{\mathbb{C}}$, the complexification of $h_{1}$, to the spectral properties of $A$ on $l_{\mathbb{C}}^{2}(\Gamma)$. Let $\langle\xi \mid Q \xi\rangle$ be the unique continuous, bilinear and strictly positive form on $\Omega$ corresponding to $\mu$ and let $\mathscr{D}\left(Q^{1 / 2}\right)$ be the closure of $\Omega$ with respect to the scalar product $\langle\xi \mid Q \xi\rangle=\langle\xi \mid \xi\rangle_{Q}$. Then $h_{1}$ is isometrically isomorphic to $\mathscr{D}\left(Q^{1 / 2}\right)$. Under this mapping $U_{1}(t)$ goes over to $T_{t}$ on $\mathscr{D}\left(Q^{1 / 2}\right)$. The generator of $T_{t}$ is the skew-adjoint operator $\left(\begin{array}{cc}0 & I \\ -A & 0\end{array}\right)$ with square

$\left(\begin{array}{ll}A & 0 \\ 0 & A\end{array}\right)$. By the stationary of $\mu,(2.6), Q$ commutes with

$$
\left(\begin{array}{ll}
A & 0 \\
0 & A
\end{array}\right)
$$

and therefore, by the spectral theorem,

$$
\left\langle\xi \mid\left(\begin{array}{cc}
A & 0 \\
0 & A
\end{array}\right) \xi\right\rangle_{Q}=\left\langle Q^{1 / 2} \xi \mid\left(\begin{array}{cc}
A & 0 \\
0 & A
\end{array}\right) Q^{1 / 2} \xi\right\rangle
$$

on $\Omega$. Since $\langle\xi \mid Q \xi\rangle$ is strictly positive, we can map $\mathscr{D}\left(Q^{1 / 2}\right)$ onto $l^{2}(\Gamma) \oplus l^{2}(\Gamma)$ through $\xi \rightarrow Q^{1 / 2} \xi$. Let $S_{t}$ be the image of $T_{t}$ under this mapping. Then (4.1) implies that the square of the generator of $S_{t}$ is $-\left(\begin{array}{ll}A & 0 \\ 0 & A\end{array}\right)$ on $l^{2}(\Gamma) \oplus l^{2}(\Gamma)$. Going over to the complexification of $l^{2}(\Gamma) \oplus l^{2}(\Gamma)$ proves the assertion.

The stability of equilibrium states of harmonic systems has been recently studied by Pulvirenti [24] following the work of Haag et al. [25] for quantum systems and of Aizenman et al. [26] for classical systems. We do not want to go here into the technical details but rather describe the results (somewhat unprecisely). Let 
$\mu$ be $T_{t}^{*}$-invariant. We call $\mu$ stable, if for each perturbation $\lambda f$, i.e. if $H$ is the formal Hamiltonian for $T_{t}^{*}$ then the formal Hamiltonian for the perturbed time evolution is $H+\lambda f$, there exists a stationary state $\mu_{\lambda f}$ such that:

(i) $d \mu_{\lambda f} / d \mu=\varrho_{\lambda f} \in L^{2}(\mu)$,

(ii) $\lim _{\lambda \rightarrow 0} \lambda^{-1}\left(\varrho_{\lambda f}-1\right)$ exists in $L^{2}(\mu)$,

(iii) The derivative (ii) is continuous in $f$ in the $L^{2}(\mu)$ sense.

It is shown in [24] under certain regularity conditions for the perturbation $f$ and the stationary measure $\mu$, that, if $\left(\Omega^{\prime}, T_{t}^{*}, \mu\right)$ is weakly mixing, then stable states satisfy the classical KMS condition. For harmonic systems these are precisely the equilibrium states [14]. Therefore, if $A$ has an absolutely continuous spectrum, then the only stable stationary Gaussian measures are the equilibrium measures.

\section{The Weak Coupling Limit}

There is a general belief in non-equilibrium statistical mechanics that the steady state of a system not far from equilibrium, will not sensibly depend at least in the interior of the system, on the details of the reservoirs sustaining this steady state. In particular, the steady state should not depend very much on the coupling strength $\lambda$ between the system and the reservoirs. This gives rise to the hope that through the weak coupling limit $\lambda \rightarrow 0$ one can in some sense eliminate the reservoirs obtaining thereby a steady state of a "simple" structure, but which still contains all physically relevant information. In this context we think it to be of interest to study the dependence of the stationary states obtained in Section 3 on the coupling strength between system and reservoirs: we shall actually find that the most interesting features of the steady state are lost in this limit.

Let the interaction matrix of the infinite chain to be $A_{\lambda}$,

$$
A_{\lambda}=A_{0}+\lambda A_{c},
$$

where $A_{0}$ and $A_{c}$ are as in Section 3. We assume $P_{L} A_{c} P_{R}=0$, for simplicity. As in (2.3) $A_{\lambda}$ generates the flow $T_{t}^{\lambda *}$ on $\Omega^{\prime}$ and for the initial state of the infinite system we again choose $\mu_{i}=\mu_{\beta_{L}} \otimes \varrho \otimes \mu_{\beta_{R}}$ [cf. (3.1)]. If the conditions of Theorem 2 are fulfilled, then

$$
\lim _{t \rightarrow \infty} \mu_{i} \circ T_{t}^{\lambda *}=\mu_{\lambda}
$$

exists and we want to investigate

$$
\lim _{\lambda \rightarrow 0} \mu_{\lambda}=\mu_{0}
$$

We will show that $\mu_{0}=\mu_{\beta_{L}} \otimes \varrho \otimes \mu_{\beta_{R}}$, where $\bar{\varrho}$ is the unique state invariant under the reduced time evolution of the system in the weak coupling limit. Thus, although the original problem (5.3) is a completely stationary one, the appearance of $\bar{\varrho}$ introduces a certain dynamical aspect and it is precisely this dynamical aspect we want to investigate first. 
For this purpose, let us define the action of the reduced dynamics $\alpha_{t}^{\lambda}$ on the functions of the form $\exp [i\langle P \xi \mid x\rangle]$ by projecting the time evolved function $\exp \left[i\left\langle T_{t}^{\lambda} P \xi \mid x\right\rangle\right]$ onto the system:

$$
\alpha_{t}^{\lambda}\left(e^{i\langle P \xi \mid x\rangle}\right)=\mu_{\beta_{L}} \otimes \mu_{\beta_{R}}\left(e^{i\left\langle T_{t}^{\lambda} P \xi \mid x\right\rangle}\right),
$$

$\xi \in \Omega, x \in \Omega^{\prime}$. By Fourier integration, (5.4) defines in fact the action of $\alpha_{t}^{\lambda}$ on all observables of the system. $\alpha_{t}^{\lambda}$ is the reduced dynamics in the Heisenberg picture corresponding to the initial state $\mu_{i}$ in (5.2). The time evolution $\alpha_{t}^{\lambda}$ still contains all the memory effects due to the coupling and depends therefore in a complicated way on the dynamics of the reservoirs. To simplify we now let the coupling $\lambda$ go to zero. Then the memory effects become negligible and the system will behave in a Markovian way. Of course, if we simple let $\lambda \rightarrow 0$ keeping $t$ fixed, the system evolves according to the isolated time evolution generated by $P A_{0} P$. To compensate for the weakening of the coupling we have to rescale the time in such a way that $\lambda^{2} t=\tau$ is kept fixed. Let us suppose for a moment that $A_{c}=0$. Since there is no coupling between the system and the reservoirs, on the $\tau$-time scale the system will then exhibit fast oscillations. To take care of them, we go over to the interaction picture and arrive at the desired object

$$
\gamma_{\tau}=\lim _{\lambda \rightarrow 0} \alpha_{-\lambda-2 \tau}^{0} \alpha_{\lambda-2 \tau}^{\lambda}
$$

(5.5) is called the weak coupling limit. The weak coupling limit of specific systems has been studied by Davies and by Pulè [27, 28, cf. Remark below]. The abstract theory is developed in a series of papers on Markovian master equations by Davies $[29,30]$. The physical background is very well presented by Haake [31].

To state the existence and the explicit form of the limit (5.5) we still have to define two averages (coinciding for unitary $T_{t}^{0}$ ) for (bounded) operators $K$ on $P\left(l^{2}(Z) \oplus l^{2}(Z)\right)$

$$
\begin{aligned}
K^{+} & =\lim _{T \rightarrow \infty}(2 T)^{-1} \int_{-T}^{T} d t T_{-t}^{0} K T_{t}^{0}, \\
K^{++} & =\lim _{T \rightarrow \infty}(2 T)^{-1} \int_{-T}^{T} d t T_{t}^{0 *} K T_{t}^{0} .
\end{aligned}
$$

Theorem 4. Let

$$
\begin{aligned}
& \left\|P B_{c}(1-P) T_{t}^{0}(1-P) B_{c} P\right\| \in L^{1}(R), \\
& \left\|P B_{c}^{*}(1-P)\left(Q_{L}+Q_{R}\right) T_{t}^{0}(1-P) B_{c} P\right\| \in L^{1}(R),
\end{aligned}
$$

where $B_{c}=\left(\begin{array}{cc}0 & 0 \\ -A_{c} & 0\end{array}\right)$ and where $Q_{L}$ and $Q_{R}$ are defined in (3.14). Then the weak coupling limit (5.5) exists uniformly on every finite interval $\left[0, \tau_{0}\right] . \gamma_{\tau}$ has the semigroup property $\gamma_{\tau_{1}} \gamma_{\tau_{2}}=\gamma_{\tau_{1}+\tau_{2}}$ and is given by

$$
\gamma_{\tau}\left(e^{i\langle\xi \mid x\rangle}\right)=\exp \left\{i\left\langle e^{L \tau} \xi \mid x\right\rangle-\frac{1}{2} \int_{0}^{\tau} d s\left\langle e^{L s} \xi \mid Q e^{L s} \xi\right\rangle\right\}
$$


where $\xi, x \in R^{2(N-M+1)}$ and

$$
\begin{aligned}
& L=\left[\int_{0}^{\infty} d s T_{-s}^{0} P B_{c}(1-P) T_{s}^{0}(1-P) B_{c} P\right]^{+}, \\
& Q=\left[\int_{-\infty}^{\infty} d s T_{1 / 2 s}^{0 *} P B_{c}^{*}(1-P)\left(Q_{L}+Q_{R}\right) T_{s}^{0}(1-P) B_{c} P T_{-1 / 2 s}^{0}\right]^{++} .
\end{aligned}
$$

Remark. Davies [27] uses a different initial state. In our terminology it is the Gaussian measure with covariance matrix

$$
\beta^{-1}(1-P)\left(\begin{array}{cc}
1 & 0 \\
0 & \left(A_{0}+\lambda A_{c}\right)^{-1}
\end{array}\right)(1-P) .
$$

In general, this state is not the equilibrium state at reciprocal temperature $\beta$ for the uncoupled reservoir. Accordingly Davies obtains a different semigroup $\gamma_{\tau}^{D}, \tau \geqq 0$, in the weak coupling limit. It is given by (again in our terminology and generalized to a system of arbitrary length)

$$
\gamma_{\tau}^{D}\left(e^{i\langle\xi \mid x\rangle}\right)=\exp \left\{i\left\langle e^{L \tau} \xi \mid x\right\rangle-\frac{1}{2}\left\langle\xi \mid Q_{0} \xi\right\rangle-\frac{1}{2}\left\langle e^{L \tau} \xi \mid Q_{0} e^{L \tau} \xi\right\rangle\right\}
$$

with

$$
Q_{0}=\left(\begin{array}{cc}
P & 0 \\
0 & \left(P A_{0} P\right)^{-1}
\end{array}\right)
$$

Proof. Since $\mu_{\beta_{L}}$ and $\mu_{\beta_{R}}$ are Gaussian measures the integration in (5.4) is easily performed to give

$$
\begin{aligned}
\alpha_{-t}^{0} \alpha_{t}^{\lambda}\left(e^{i\langle P \xi \mid x\rangle}\right)= & \exp \left\{i\left\langle P T_{-t}^{0} T_{t}^{\lambda} P \xi \mid x\right\rangle\right. \\
& \left.-\frac{1}{2}\left\langle(1-P) T_{t}^{\lambda} P \xi \mid\left(Q_{L}+Q_{R}\right)(1-P) T_{t}^{\lambda} P \xi\right\rangle\right\} .
\end{aligned}
$$

If we study $P T_{-t}^{0} T_{t}^{\lambda} P$ in $l^{2}(Z) \oplus l^{2}(Z)$, we have the same abstract setting as in [29]. $\left(T_{t}^{\lambda}\right.$ is not a group of isometries. For the case at hand this is not needed.) By (5.8) we can apply Theorem 2.2 of [29] asserting the existence of

$$
\lim _{\lambda \rightarrow 0} P T_{-\lambda-2 \tau}^{0} T_{\lambda-2 \tau}^{\lambda} P=e^{L \tau}
$$

uniformly on every finite interval $\left[0, \tau_{0}\right]$ with $L$ as in (5.10). Let us denote the second term in the exponent by $r^{\lambda}(t)$. We note the identity

$$
(1-P) T_{t}^{\lambda} P=\lambda \int_{0}^{t} d s T_{t-s}^{0}(1-P) B_{c} P T_{s}^{\lambda} P .
$$

Inserting (5.15) on both sides in (5.13) we obtain

$$
\begin{aligned}
r^{\lambda}\left(\lambda^{-2} \tau\right)= & \lambda^{-2} \int_{0}^{\tau} d \sigma \int_{0}^{\tau} d \sigma^{\prime}\left\langle P T_{\lambda-2 \sigma}^{\lambda} P \xi\right| P B_{c}^{*}(1-P)\left(Q_{L}+Q_{R}\right) \\
& \left.\cdot T_{\lambda-2\left(\sigma-\sigma^{\prime}\right)}^{0}(1-P) B_{c} P T_{\lambda-2 \sigma^{\prime}}^{\lambda} P \xi\right\rangle .
\end{aligned}
$$


Changing the integration to $\lambda^{2} y=\sigma-\sigma^{\prime}, y=\frac{1}{2}\left(\sigma+\sigma^{\prime}\right)$ and choosing a basis $\left|\xi_{k}\right\rangle$ in $P\left(l^{2}(Z) \oplus l^{2}(Z)\right)$

$$
\begin{aligned}
& r^{\lambda}\left(\lambda^{-2} \tau\right)=\sum_{k, j} \int_{-\lambda^{-2} \tau}^{\lambda^{2} \tau} d y\left\langle\xi_{k}\right| P T_{1 / 2 y}^{0 *} P B_{c}^{*}(1-P)\left(Q_{L}+0_{R}\right) T_{y}^{0} \\
& \left.(1-P) B_{c} P T_{-1 / 2 y}^{0} \xi_{j}\right\rangle \int_{1 / 2 \lambda^{2}|y|}^{\tau-1 / 2 \lambda^{2}|y|} d y^{\prime}\left\langle P T_{-1 / 2 y-\lambda^{-2} y^{\prime}}^{0} T_{1 / 2 y+\lambda^{-2} y^{\prime}}^{\lambda} P \xi\right| \\
& \left.\left.\left(T_{\lambda^{-2} y^{\prime}}^{0 *} \xi_{k}\right\rangle\left\langle\xi_{j}\right| T_{\lambda^{-2} y^{\prime}}^{0}\right) P T_{1 / 2 y-\lambda^{-2} y^{\prime}}^{0} T_{-1 / 2 y+\lambda^{-2} y^{\prime}}^{\lambda} P \xi\right\rangle .
\end{aligned}
$$

On $[0, \tau] P T_{-1 / 2 y-\lambda-2 y^{\prime}}^{0} \quad T_{1 / 2 y+\lambda-2 y^{\prime}}^{\lambda} P \xi$ converges uniformly to $e^{L y^{\prime}} \xi$. Therefore the second integral converges to

$$
\int_{0}^{\tau} d y^{\prime}\left\langle e^{L y^{\prime}} \xi \mid\left(\left|\xi_{k}\right\rangle\left\langle\xi_{j}\right|\right)^{++} e^{L y^{\prime}} \xi\right\rangle
$$

By assumption the first term is in $L^{1}$. Since the second integral is bounded, by Lebesgue's dominated convergence theorem $r^{\lambda}\left(\lambda^{-2} \tau\right)$ converges as $\lambda \rightarrow 0$ to the expression given in (5.9).

Equation (5.9) can be converted to the more familiar differential form. With an obvious notation we obtain

$$
\begin{aligned}
\partial f(p, q) / \partial t= & \{\langle(p, q) \mid L(\partial / \partial p, \partial / \partial q)\rangle \\
& +1 / 2\langle(\partial / \partial p, \partial / \partial q) \mid Q(\partial / \partial p, \partial / \partial q)\rangle\} f(p, q) .
\end{aligned}
$$

This is a Fokker-Planck type differential equation. It should be noted that the solutions of the differential equation (5.9) do not necessarily decay. Modes of the system with frequencies not in the band of the uncoupled reservoir are not damped and will therefore oscillate with a, in general, modified frequency (cf. Sect. 6).

After this excursion, let us return to the original problem (5.3). As already for the weak coupling limit, the existence of the limit (5.3) will be ensured by a sufficient fast decay of functions of the form $\left\langle\eta \mid T_{t}^{\lambda} \xi\right\rangle$. Here we need even stronger conditions than in (5.8). The essential new condition (5.21) expresses a kind of uniform behavior of $\left\langle\eta \mid T_{t}^{\lambda} \xi\right\rangle$ for small $\lambda$ 's. (5.21) implies then the uniform convergence of the weak coupling limit, the existence of a unique stationary state $\bar{\varrho}$ of the semigroup $\gamma_{\tau}$ and the approach to stationarity: $\lim _{t \rightarrow \infty} \gamma_{\tau}^{*}(\varrho)=\bar{\varrho}$ for every initial state $\varrho$. Conditions (5.8), (5.20), and (5.21) are hard to prove in general. A simple case will be discussed in the next section (cf. also [27], where related conditions are proved).

Theorem 5. Let $A_{0}$ be bounded away from zero. For given $\eta, \xi \in \Omega$, let

$$
\begin{aligned}
& \left\langle P \eta \mid B_{c}(1-P) T_{t}^{0} \xi\right\rangle \in L^{1}(R) \\
& \left\langle P \eta \mid B_{c}^{*}(1-P)\left(Q_{L}+Q_{R}\right) T_{t}^{0} \xi\right\rangle \in L^{1}(R),
\end{aligned}
$$

and

$$
\left|\left\langle P \eta \mid T_{t}^{\lambda} \xi\right\rangle\right| \leqq f\left(\lambda^{2} t\right) \in L^{2}(R)
$$

for all $\lambda \in(0, \bar{\lambda}], \bar{\lambda}>0$. Then

(i) $\lim _{t \rightarrow \infty}\left(\mu_{\beta_{L}} \otimes \varrho \otimes \mu_{\beta_{R}}\right) \circ T_{t}^{\lambda *}=\mu_{\lambda}$ 
exists in the weak sense, independently of $\varrho$, for all $\lambda \in\left(0, \lambda_{0}\right], 0<\lambda_{0} \leqq \bar{\lambda} . \mu_{\lambda}$ is a Gaussian measure on $\Omega^{\prime}$.

$$
\text { (ii) } \lim _{\lambda \rightarrow 0} \mu_{\lambda}=\mu_{\beta_{L}} \otimes \varrho \unrhd \mu_{\beta_{R}}
$$

exists in the weak sense. $\mu_{\beta_{L}} \otimes \varrho \otimes \mu_{\beta_{R}}$ is a Gaussian measure on $\Omega^{\prime}$, where $\underline{\varrho}$ is the unique stationary state of the semigroup $\gamma_{\tau}$ in $(5.9)$ (i.e. $\gamma_{\tau}^{*}(\bar{\varrho})=\bar{\varrho}$ for all $\tau \geqq 0$ ).

Proof. We study the limits of the respective Fourier transforms. As in (3.15) the Fourier transform of $\left(\mu_{\beta_{L}} \otimes \varrho \otimes \mu_{\beta_{R}}\right) \circ T_{t}^{\lambda *}$ is

$$
\varrho\left(e^{i\left\langle P T_{t}^{\lambda} \xi \mid \cdot\right\rangle}\right) \exp \left\{-1 / 2\left\langle T_{t}^{\lambda} \xi \mid\left(Q_{L}+Q_{R}\right) T_{t}^{\lambda} \xi\right\rangle\right\} .
$$

By (5.21) $\lim _{t \rightarrow \infty}\left\langle P T_{t}^{\lambda} \xi \mid x\right\rangle=0$. By (5.15) the second term can be rewritten as

$$
\begin{aligned}
\langle\xi|\left(Q_{L}\right. & \left.\left.+Q_{R}\right) \xi\right\rangle+\lambda \int_{0}^{t} d s\left\langle\xi \mid\left(Q_{L}+Q_{R}\right) T_{-s}^{0}(1-P) B_{c} P T_{s}^{\lambda} \xi\right\rangle \\
& +\lambda \int_{0}^{t} d s\left\langle P T_{s}^{\lambda} \xi \mid B_{c}^{*}(1-P)\left(Q_{L}+Q_{R}\right) T_{-s}^{0} \xi\right\rangle \\
& +\lambda^{2} \int_{0}^{t} d s^{\prime} \int_{0}^{t} d s\left\langle T_{s}^{\lambda} \xi \mid P B_{c}^{*}(1-P)\left(Q_{L}+Q_{R}\right) T_{s-s^{\prime}}^{0}(1-P) B_{c} P T_{s^{\prime}}^{\lambda} \xi\right\rangle .
\end{aligned}
$$

Since $A_{0}$ is bounded away from zero, $A_{0}+\lambda A_{c}$ is also bounded away from zero for 0 $\leqq \lambda \leqq \lambda_{0} \leqq \bar{\lambda}$. Therefore, for $\lambda \in\left[0, \lambda_{0}\right],\left\langle P \eta \mid T_{t}^{\lambda} \xi\right\rangle$ is bounded. By (5.20), the integrand of the first integral in (5.25) is integrable. This ensures the existence of the limit as $t \rightarrow \infty$. The same argument applies to the second integral. For the third integral in (5.25) we use the same method as in (5.17). By (5.20) the first integrand and by (5.21) the second integrand of that integral is integrable on $R$. Thus, for $\lambda \in\left(0, \lambda_{0}\right]$, the Fourier transform of $\left(\mu_{\beta_{L}} \otimes P \otimes \mu_{\beta_{R}}\right) \circ T_{t}^{\lambda *}$ converges as $t \rightarrow \infty$ to

$$
\begin{aligned}
& \left\langle(1-P) \xi \mid\left(Q_{L}+Q_{R}\right)(1-P) \xi\right\rangle+\lambda \int_{0}^{\infty} d s\left\langle\xi \mid\left(Q_{L}+Q_{R}\right) T_{-s}^{0}(1-P) B_{c} P T_{s}^{\lambda} \xi\right\rangle \\
& +\lambda \int_{0}^{\infty} d s\left\langle P T_{s}^{\lambda} \xi \mid B_{c}^{*}(1-P)\left(Q_{L}+Q_{R}\right) T_{-s}^{0} \xi\right\rangle \\
& +\sum_{k, j} \int_{-\infty}^{\infty} d y\left\langle P \xi_{k} \mid T_{1 / 2 y}^{0 *} P B_{c}^{*}(1-P)\left(Q_{L}+Q_{R}\right) T_{y}^{0}(1-P) B_{c} P T_{-1 / 2 y}^{0} P \xi_{j}\right\rangle \\
& \quad \cdot \int_{1 / 2 \lambda^{2}|y|}^{\infty} d y^{\prime}\left\langle P T_{-1 / 2 y-\lambda^{-2} y^{\prime}}^{0} T_{1 / 2 y+\lambda^{-2} y^{\prime}}^{\lambda} \xi\right|\left(T_{\lambda^{-2} y^{\prime}}^{0 *} \xi_{k}\right\rangle \\
& \left.\left.\quad \cdot\left\langle\xi_{0}\right| T_{\lambda^{-2} y^{\prime}}^{0}\right) P T_{1 / 2 y-\lambda^{-2} y^{\prime}}^{0} T_{-1 / 2 y+\lambda^{-2} y^{\prime}}^{\lambda} \xi\right\rangle .
\end{aligned}
$$

(5.26) is a continuous, bilinear and strictly positive form on $\Omega$ defining the Gaussian measure $\mu_{\lambda}$.

We show that the semigroup $\left\{\gamma_{\tau} \mid \tau \geqq 0\right\}$ in (5.9) has a unique stationary state. Suppose that $L$ has an eigenvalue $x$ with $\operatorname{Re}(x) \geqq 0$ and let $P \xi$ be the corresponding eigenvector. By (5.21) we can find, given $0<\varepsilon<\frac{1}{2}$, a $\tau_{0}$ such that for all $\tau \geqq \tau_{0}$

$$
\left|\left\langle T_{-\lambda-2_{\tau}}^{0} T_{\lambda-{ }_{\tau} \tau}^{\lambda} P \xi \mid P \xi\right\rangle\right|<\varepsilon
$$


for $\lambda \leqq \lambda_{0}$. We can choose a $\tau^{\prime}>\tau_{0}$ such that $\left|\left\langle e^{L \tau^{\prime}} P \xi \mid P \xi\right\rangle\right| \geqq 1$. However by (5.14)

$$
\lim _{\lambda \rightarrow 0}\left\langle P T_{-\lambda^{-2} \tau}^{0} T_{\lambda-{ }_{2} \tau}^{\lambda} P \xi \mid P \xi\right\rangle=\left\langle e^{L \tau} P \xi \mid P \xi\right\rangle
$$

uniformly on $\left[0, \tau^{\prime}\right]$ which contradicts (5.27). Therefore the spectrum of $L$ lies in the open left hand plane. By (5.9) this implies

$$
\lim _{\tau \rightarrow \infty} \gamma_{\tau}^{*}(\varrho)=\bar{\varrho}
$$

for arbitrary initial states $\varrho . \varrho$ is the Gaussian measure with covariance

$$
\int_{0}^{\infty} d s\left\langle e^{L s} P \xi \mid Q e^{L s} P \xi\right\rangle .
$$

We investigate the limit as $\lambda \rightarrow 0$ of the quadratic form (5.26). Since $\lim _{\lambda \rightarrow 0}\left\{\sup _{t}\left|\lambda\left\langle P \eta \mid T_{t}^{\lambda} \xi\right\rangle\right|\right\}=0$, the first and the second integral converge to zero as $\lambda \rightarrow 0$. To discuss the third integral, we show that

$$
\lim _{\lambda \rightarrow 0} P T_{-\lambda-2_{\tau}}^{0} T_{\lambda-2_{\tau}}^{\lambda} \xi=e^{L \tau} P \xi
$$

uniformly on $[0, \infty)$. Since $L$ is strictly contracting and by (5.21), given $\varepsilon>0$, we can choose a $\tau_{0}$ such that for all $\tau>\tau_{0}$

$$
\left\|e^{L \tau} P \xi\right\|<\varepsilon / 2,\left\|P T_{-\lambda-2_{\tau}}^{0} T_{\lambda^{-2} \tau}^{\lambda} P \xi\right\|<\varepsilon / 2
$$

for $\lambda<\bar{\lambda}$. In contradistinction to the weak coupling limit in Theorem 4 , we have now $\xi \in \Omega$. As in $[29, \S 2]$ we obtain

$$
\begin{aligned}
& P T_{-\lambda-z_{\tau}}^{0} T_{\lambda^{-2} \tau}^{\lambda} \xi=P \xi+\lambda \int_{0}^{\lambda-2} d s T_{-s}^{0} P B_{c}(1-P) T_{s}^{0} \xi \\
& +\int_{\sigma=0}^{\tau} d \sigma T_{-\lambda_{-2} \sigma}^{0}\left[\int_{s=0}^{\lambda-2(\tau-\sigma)} d s T_{-s}^{0} P B_{c}(1-P) T_{s}^{0}(1-P) B_{c} P\right] \\
& \cdot T_{\lambda-\lambda_{\sigma}}^{0} P T_{-\lambda^{-2} \sigma}^{0} T_{\lambda^{-2} \sigma}^{\lambda} \xi .
\end{aligned}
$$

By (5.20)

$$
\left\|\lambda \int_{0}^{\lambda-2 \tau} d s T_{-s}^{0} P B_{c}(1-P) T_{s}^{0} \xi\right\| \leqq \lambda c \int_{0}^{\infty} d s\left\|P B_{c}(1-P) T_{s}^{0} \xi\right\| .
$$

Therefore, by the same proof as the one of [29, Theorem 2.1], we conclude that

$$
\lim _{\lambda \rightarrow 0} P T_{-\lambda-2_{\tau}}^{0} T_{\lambda^{-2} \tau}^{\lambda} \xi=e^{L \tau} P \xi
$$

uniformly on $\left[0, \tau_{0}\right]$. Putting (5.35) and (5.32) together proves (5.31). Using (5.31) we conclude as in (5.18) that the fourth term in (5.26) converges to

$$
\int_{0}^{\infty} d s\left\langle e^{L s} P \xi \mid Q e^{L s} P \xi\right\rangle .
$$

Therefore the Fourier transform of $\lim _{\lambda \rightarrow 0} \mu_{\lambda}$ is

$$
\exp \left\{-\frac{1}{2}\left[\left\langle(1-P) \xi \mid\left(Q_{L}+Q_{R}\right)(1-P) \xi\right\rangle+\int_{0}^{\infty} d s\left\langle e^{L s} P \xi \mid Q e^{L s} P \xi\right\rangle\right]\right\} .
$$




\section{Rubin's Model Revisited}

In this model the left and right reservoirs are chosen to consist of unit masses with nearest neighbor coupling of unit strength [9]. The reservoirs are coupled to the first and last particle of the system, respectively, with strength $\lambda$. There is no restriction on the harmonic forces between the particles of the system. The particular simple form of the reservoirs allows one to check the condition (5.8) explicitly and to evaluate the averages (5.6) and (5.7) in terms of the normal modes of the system. Hopefully, this section serves as an illustration of the results of the preceding section and provides also a link to the detailed information about open harmonic systems obtained by other methods.

Let $M=1$ and let

$$
P A P=\sum_{j=1}^{N} \omega_{j}^{2}\left|\xi_{j}\right\rangle\left\langle\xi_{j}\right|,
$$

where we assume, for simplicity of notation, that the eigenfrequencies $\omega_{j}>0$ are all different from each other. The conditions (5.8) of Theorem 4 lead to the following two integrals

$$
\int_{0}^{2}\left(4-x^{2}\right)^{1 / 2} x \sin (x t) d x, \int_{0}^{2}\left(4-x^{2}\right)^{1 / 2} \cos (x t) d x .
$$

Both functions are in $L^{1}(R)$. Therefore the weak coupling limit exists. Evaluating the averages (5.6) and (5.7) one obtains

$$
L=-\left(\begin{array}{cc}
L_{1} & L_{2} \\
-P A P L_{2} & L_{1}
\end{array}\right)
$$

with

$$
\begin{aligned}
L_{1}= & \frac{1}{4} \sum_{\omega_{j}<2}\left(\xi_{j, 1}^{2}+\xi_{j, N}^{2}\right)\left(4-\omega_{j}^{2}\right)^{1 / 2}\left|\xi_{j}\right\rangle\left\langle\xi_{j}\right| \\
L_{2}= & \frac{1}{4} \sum_{\omega_{j}<2}\left(\xi_{j, 1}^{2}+\xi_{j, N}^{2}\right)\left(2-\omega_{j}^{2}\right) \omega_{j}^{-2}\left|\xi_{j}\right\rangle\left\langle\xi_{j}\right| \\
& +\frac{1}{4} \sum_{2 \leqq \omega_{j}}\left(\xi_{j, 1}^{2}+\xi_{j, N}^{2}\right) \omega_{j}^{-2}\left(\left(2-\omega_{j}^{2}\right)+\omega_{j}\left(\omega_{j}^{2}-4\right)^{1 / 2}\right)\left|\xi_{j}\right\rangle\left\langle\xi_{j}\right|
\end{aligned}
$$

and

$$
Q=\left(\begin{array}{cc}
Q_{l}+Q_{r} & 0 \\
0 & (P A P)^{-1}\left(Q_{l}+Q_{r}\right)
\end{array}\right)
$$

with

$$
\begin{aligned}
& Q_{l}=\left(2 \beta_{L}\right)^{-1} \sum_{\omega_{j}<2}\left(4-\omega_{j}^{2}\right)^{1 / 2} \xi_{j, 1}^{2}\left|\xi_{j}\right\rangle\left\langle\xi_{j}\right| \\
& Q_{r}=\left(2 \beta_{R}\right)^{-1} \sum_{\omega_{j}<2}\left(4-\omega_{j}^{2}\right)^{1 / 2} \xi_{j, N}^{2}\left|\xi_{j}\right\rangle\left\langle\xi_{j}\right| .
\end{aligned}
$$


The solution of the equations

$$
\frac{d}{d t}\left(\begin{array}{l}
p \\
q
\end{array}\right)=\left(\begin{array}{cc}
L_{1} & L_{2} \\
-P A P L_{2} & L_{1}
\end{array}\right)\left(\begin{array}{l}
p \\
q
\end{array}\right)
$$

reads

$$
\left(\begin{array}{l}
p(t) \\
q(t)
\end{array}\right)=\left(\begin{array}{l}
\sum_{j} e^{-\lambda j^{t}}\left[\cos \left(k_{j} t\right)\left|\xi_{j}\right\rangle\left\langle\xi_{j} \mid p\right\rangle-\omega_{j}^{-1} \sin \left(k_{j} t\right)\left|\xi_{j}\right\rangle\left\langle\xi_{j} \mid q\right\rangle\right] \\
\sum_{j} e^{-\lambda} j^{t}\left[\omega_{j} \sin \left(k_{j} t\right)\left|\xi_{j}\right\rangle\left\langle\xi_{j} \mid p\right\rangle+\cos \left(k_{j} t\right)\left|\xi_{j}\right\rangle\left\langle\xi_{j} \mid q\right\rangle\right]
\end{array}\right),
$$

where

$$
\begin{aligned}
\lambda_{j} & =\left\{\begin{array}{l}
\frac{1}{4}\left(\xi_{j, 1}^{2}+\xi_{j, N}^{2}\right)\left(4-\omega_{j}^{2}\right)^{1 / 2}, \omega_{j}<2 \\
0, \omega_{j} \geqq 2
\end{array}\right. \\
k_{j} & =\left\{\begin{array}{l}
\frac{1}{4}\left(\xi_{j, 1}^{2}+\xi_{j, N}^{2}\right) \omega_{j}^{-1}\left(2-\omega_{j}^{2}\right), \omega_{j}<2 \\
\frac{1}{4}\left(\xi_{j, 1}^{2}+\xi_{j, N}^{2}\right)\left[\omega_{j}^{-1}\left(2-\omega_{j}^{2}\right)+\left(\omega_{j}^{2}-4\right)^{1 / 2}\right], \omega_{j} \geqq 2 .
\end{array}\right.
\end{aligned}
$$

Thus, as expected, all frequencies in the band of the reservoir $(0<\omega<2)$ get damped, provided that $\xi_{j, 1}^{2}+\xi_{j, N}^{2} \neq 0$, whereas all modes with frequencies outside the band oscillate with some modified frequency. This property carries over immediately to the time evolution of observables. If all eigenvalues $\omega_{j}^{2}$ of the interaction matrix $P A P$ are smaller than 4 and if $\xi_{j, 1}^{2}+\xi_{j, N}^{2} \neq 0$ for all $j=1, \ldots, N$, then every initial phase function will converge to a multiple of the constant function. In terms of the Schrödinger picture, every initial state will converge as $\tau \rightarrow \infty$ to a unique stationary state (dependent on the reservoir temperatures). This unique stationary state is a Gaussian measure on $R^{2 N}$. Its covariance matrix is given by

$$
\left(\begin{array}{cc}
Q_{s} & 0 \\
0 & (P A P)^{-1} Q_{s}
\end{array}\right)
$$

where $Q_{s}$ is a function of $P A P$ given by

$$
\begin{aligned}
Q_{s} & =\sum_{j=1}^{N} \frac{T_{L} \xi_{j, 1}^{2}+T_{R} \xi_{j, N}^{2}}{\xi_{j, 1}^{2}+\xi_{j, N}^{2}}|\xi\rangle\left\langle\xi_{j}\right|, \\
T_{L} & =\beta_{L}^{-1}, T_{R}=\beta_{R}^{-1}
\end{aligned}
$$

Obviously, for $T_{L}=T_{R}$ we obtain the covariance matrix of the canonical ensemble.

The stationary heat flow through the system is defined as the energy flux function from the left reservoir to the system averaged over the stationary state given by (6.7). Here, the energy flux function is the change of the energy of the system due to the coupling with the left reservoir. [We could, of course, compute the same quantity with the right reservoir. Since the total heat flow is zero in the 
stationary state, we would obtain then the negative of (6.11).] For the energy flux function $J$ we find

$$
J(p, q)=\left\langle(p, q)\left\langle\left(\begin{array}{cc}
-L_{1} & -P A P L_{2} \\
P A P L_{2} & -P A P L_{1}
\end{array}\right)_{\mathrm{left}}(p, q)\right\rangle+\operatorname{tr} Q_{l} .\right.
$$

The index "left" reminds us that we have to take in (6.3) only those terms coming from the coupling to the left reservoir, i.e. in (6.3) we have to set $\xi_{j, N}^{2}=0$. Evaluating the average, we obtain the heat flow

$$
\langle J\rangle=\left(T_{L}-T_{R}\right) \frac{1}{2} \sum_{j=1}^{N} \frac{\xi_{j, 1}^{2} \cdot \xi_{j, N}^{2}}{\xi_{j, 1}^{2}+\xi_{j, N}^{2}}\left(4-\omega_{j}^{2}\right)^{1 / 2} .
$$

It should be noted that up to the frequency cut off factor $\left(4-\omega_{j}^{2}\right)^{1 / 2},(6.11)$ is identical to the heat flow through a harmonic chain weakly coupled to stochastic reservoirs (Lebowitz's model). The kinetic temperature $T_{n}$ of the $n$-th particle is given by

$$
T_{n}=\left\langle p_{n}^{2}\right\rangle=\sum_{j=1}^{N} \frac{T_{L} \xi_{j, 1}^{2}+T_{R} \xi_{j, N}^{2}}{\xi_{j, 1}^{2}+\xi_{j, N}^{2}} \xi_{j, n}^{2} .
$$

How do these results compare to that obtained for finite coupling $\lambda$ ? To answer that question, we specialize the system to nearest neighbor coupling of unit strength and arbitrary masses $m_{j} \geqq 1$. We can then follow step by step the derivation in $[4, \S$ 2], where the heat flow was computed for $\lambda=1$. Let $Y_{N}(\omega, \lambda)=A-M \omega^{2}-U_{N} \lambda^{2} A(\omega)$, where $A$ is a tridiagonal matrix with $A_{i i}=2, A_{i j}=-1$, if $|i-j|=1, A_{i j}=0$ otherwise, $M$ is a diagonal matrix with $M_{j j}=m_{j}, U_{N}$ is a diagonal matrix with $\left(U_{N}\right)_{j j}=\delta_{j 1}+\delta_{j N}$ and $A(\omega)=\frac{1}{2}\left[2-\omega^{2}-i \omega\left(4-\omega^{2}\right)^{1 / 2}\right]$. Let $\Delta_{i, j}(\omega, \lambda)$ be defined as the determinant of the submatrix of $Y_{N}(\omega, \lambda)$ beginning with the $i$-th row and column ending at the $j$-th row and column and let $\Delta_{1,0}(\omega, \lambda)=1=\Delta_{N+1, N}(\omega, \lambda)$. For the heat flow one obtains then

$$
J(\lambda)=\frac{1}{4 \pi}\left(T_{L}-T_{R}\right) \lambda^{4} \int_{0}^{2} \omega^{2}\left(4-\omega^{2}\right)\left|\Delta_{1, N}(\omega, \lambda)\right|^{-2} d \omega .
$$

For $\lambda=1$, this agrees with $[4,(2.16)]$. For the kinetic temperature one obtains

$$
\begin{aligned}
T_{j}(\lambda)= & (4 \pi)^{-1} m_{j} \lambda^{2} \int_{0}^{2} \omega^{2}\left(4-\omega^{2}\right)^{1 / 2}\left|\Delta_{1, N}(\omega, \lambda)\right|^{-2}\left(T_{L}\left|\Delta_{j+1, N}(\omega, \lambda)\right|^{2}\right. \\
& \left.+T_{R}\left|\Delta_{1, j-1}(\omega, \lambda)\right|^{2}\right) d \omega .
\end{aligned}
$$

For $\lambda=1$, this agrees with the result of Rubin and Greer [9, (3.15)], who computed the kinetic temperature for $j=1$ and $j=N$ by a rather different method.

As $\lambda \rightarrow 0, \Delta_{1, N}(\omega, \lambda)$ becomes singular at the eigenvalues $\omega_{j}$. By a similar technique as in $[4, \S 5]$ one shows that the temperature $T_{j}(\lambda)$ goes over to the value $T_{j}$ given in (6.12). The heat flow decreases as the coupling becomes weaker. Therefore $J(\lambda)$ vanishes as $\lambda \rightarrow 0$. For the lowest order contribution in $\lambda$ one obtains, again by the same technique as in $[4, \S 5]$,

$$
\lim _{\lambda \rightarrow 0} \lambda^{-2} J(\lambda)=\langle J\rangle,
$$

where $\langle J\rangle$ is the average heat flow (6.11) in the weak coupling limit. 
The appearance of $\lambda^{-2}$ can be understood by expanding the stationary state $\mu_{\lambda}$ as

$$
\mu_{\lambda}=\mu_{\beta_{L}} \otimes \varrho \varrho \otimes \mu_{\beta_{R}}+\lambda \mu_{1}+\lambda^{2} \mu_{2}+\ldots
$$

The energy flux function is $\lambda q_{0} p_{1}$. Therefore

$$
J(\lambda)=\mu_{\lambda}\left(\lambda q_{0} p_{1}\right)=\lambda^{2} \mu_{1}\left(q_{0} p_{1}\right)+\ldots
$$

since by (6.8) the first term in the average vanishes. Alternatively, one could choose as energy flux function e.g. $q_{1} p_{2}$. Then the average over $\mu_{2}$ would be the first nonvanishing contribution to the heat flow. The reason that the lowest order in $\lambda$ is $\langle J\rangle$ is somewhat more subtle. We look at the average change of energy $\mu_{\lambda}\left(H(t)_{\text {left }}\right)$ due to the coupling to the left reservoir. Then $\mu_{\lambda}\left(H\left(\lambda^{-2} \tau\right)_{\text {left }}\right) \rightarrow\left\langle H(\tau)_{\text {left }}\right\rangle$ as $\lambda \rightarrow 0$. Here one uses that for small $\lambda$ the interaction representation can be "absorbed" by the stationary state $\mu_{\lambda}$. If the derivatives also converge,

$$
\begin{aligned}
& \frac{d}{d \tau} \mu_{\lambda}\left(H\left(\lambda^{-2} \tau\right)_{\text {left }}\right)=\lambda^{-2} \mu_{\lambda}\left(\frac{d}{d t} H(t)_{\text {left }}\right)=\lambda^{-2} J(\lambda) \\
& \rightarrow\left\langle\frac{d}{d \tau} H(\tau)_{\text {left }}\right\rangle=\langle J\rangle
\end{aligned}
$$

as $\lambda \rightarrow 0$, then one obtains $(6.15)$.

\section{Concluding Remarks}

i) In this paper we exploited the essential simplicity of harmonic systems: the linearity of their equations of motion, stemming from the fact that the Hamiltonian is quadratic in the dynamical variables. This linearity, and not so much the classical character of the system considered, is the ingredient of all proofs. Therefore most of our results (with the exception of those in Sect. 4) carry over to quantum lattices. In both the classical and quantum case one has a space $\Omega$ of rapidly decreasing sequences and a group $T_{t}$ of linear transformations on $\Omega$. One then builds, over $\Omega$, a classical phase space $\Omega^{\prime}$, a $C C R$ algebra or a $C A R$ algebra, respectively. The time evolution $\alpha_{t}$ is induced by linearity $: \alpha_{t}:\langle\xi \mid x\rangle \rightarrow\left\langle T_{t} \xi \mid x\right\rangle, \xi \in \Omega$. For Bose lattices, one chooses on physical grounds, the same $T_{t}$ as in the classical case. Our results for the classical system have then only to be translated into another "language". For Fermi lattices, however, in order to make the time evolution compatible with the $C A R$ one has to choose, in general, a group $T_{t}$ of transformations different from the classical ones [32]. Hence to obtain analogous results for Fermi lattices requires further study.

ii) The results of Sections 2-5 can be readily generalized to higher dimensional harmonic crystals, at least in the case where the "system" is finite. We have not investigated the case of an infinite "system" coupled to infinite reservoirs.

iii) The type of stationary states investigated here for the harmonic systems are clearly not possible in more realistic systems, e.g. in anharmonic crystals in which Fourier's law is obeyed. Starting such an infinite system in an initial state $\mu_{i}$, of the form described in Section 3, we would expect, at least when the temperature 
difference between the "reservoirs" is small, that $\mu_{i} \circ T_{t}$ will approach, as $t \rightarrow \infty$, a Gibbsian equilibrium state $\mu_{\beta}$. The proper $\beta$ could presumably be determined from a solution of the heat conduction equation. The heat flux in the "middle part" of this system, $J(t)$, will then approach zero as $t \rightarrow \infty$. We expect however that the "state" of the middle part, $\varrho(t)=P\left(\mu_{i} \circ T_{t}\right) P$, will have the asymptotic (large $t$ ) behavior, $\varrho(t) \cong \varrho_{\beta}$ $+\varrho_{1} \nabla T(t)_{t}$, where $\varrho_{\beta}=P \mu_{\beta} P$ and $\nabla T(t)$ is the "temperature gradient" which goes to zero as $t \rightarrow \infty$. The temperature would be defined in terms of the local energy or kinetic energy density $[1,33]$. To be a bit more precise the limit $t \rightarrow \infty$ of $[\varrho(t)$ $\left.-\varrho_{\beta}\right] / V T(t)$ ought to exist and determine a linear functional $\varrho_{1}$ on the local algebra of the system. This functional $\varrho_{1}$ would then yield the distribution function of a system in which heat is flowing. (This is what one obtains, for the one particle distribution function, from a Chapman-Enskog type solution of the inhomogeneous Boltzmann equation.)

An alternative way of obtaining $\varrho_{1}$ would be to couple the anharmonic system to stochastic reservoirs $[2,33]$ at reciprocal temperatures $\beta_{L}$ and $\beta_{R}$. Let $\mu_{s}$ be the stationary state of this system. We could then find the limit $\mu_{1}=\left(\mu_{s}-\mu_{\beta}\right) \mathscr{L} /\left(\beta_{R}^{-1}\right.$ $-\beta_{L}^{-1}$ ), as $\beta_{L}$ and $\beta_{R}$ approach $\beta$ from opposite sides, where $\mathscr{L}$ is the length of the system. For large $\mathscr{L}$ the linear functionals $\mu_{1}$ and $\varrho_{1}$ should then become equal.

Acknowledgments. We would like to thank S. Goldstein for valuable comments and J. L. van Hemmen for a careful reading of the manuscript. For one of us (H.S.) it is a pleasure to thank the Belfer Graduate School of Science for very friendly hospitality.

\section{References}

1. Peierls, R.E.: Quantum theory of solids, Sections 2.4.-2.7. Oxford: University Press 1956 Ashcroft, N.W., Mermin, N. D. : Solid state physics, Chapter 25. New York-London: Holt, Rinehart, and Winston 1976

2. Rieder,Z., Lebowitz,J.L., Lieb,E. : J. Math. Phys. 8, 1073 (1967)

3. Casher, A., Lebowitz,J.L.: J. Math. Phys. 12, 1701 (1971)

4. O’Connor, A.J., Lebowitz,J.L.: J. Math. Phys. 15, 692 (1974)

5. Nakazawa,H.: Prog. Theor. Phys. 39, 236 (1968)

6. Bolsterli,M., Rich,M., Visscher,W.M.: Phys. Rev. A 1, 1086 (1970) Visscher,W.M. : In: Methods in computational physics. New York: Academic Press 1976

7. Matsuda,H., Ishii, K.: Supl. Prog. Theor. Phys. 45, 56 (1970)

8. Papanicolaou, G.C.: Heat conduction in a one-dimensional random lattice. Preprint 1976

9. Rubin, R.J., Greer,W.L.: J. Math. Phys. 12, 1686 (1971)

10. Klein, G., Prigogine, I. : Physica 19, 74, 89, 1053 (1953) Brout, R., Prigogine, I. : Physica 22, 621 (1956)

11. Hemmer,P.C.: Danymic and stochastic types of motion in the linear chain. Det. Fysiske Seminar i Trondheim, No. 2 (1959)

12. Lanford III,O.E., Lebowitz,J.L.: Time evolution and ergodic properties of harmonic systems. In: Lecture Notes in Physics, Vol. 38, pp. 144-177. Berlin-Heidelberg-New York: Springer 1975

13. Titulaer, U.M.: Physica 70, 257, 276, 456, (1973)

14. Hemmen,J.L.van: Dynamics and ergodicity of the infinite harmonic crystal. Thesis, University of Groningen, 1976

15. Lanford III,O.E.: Acta Physica Austr. Suppl. X, 589 (1973)

16. Goldstein,S., Lebowitz,J.L., Aizenman,M.: In: Dynamical systems - theory and applications. Lecture Notes in Physics, Vol. 38. Berlin-Heidelberg-New York: Springer 1975

17. Robinson, D., Lanford III,O.E.: Commun. math. Phys. 24, 193 (1972)

18. Goldstein,S., Lebowitz,J.L.: Commun. math. Phys. 37, 1 (1974)

19. Goldstein, S.: Thesis, Yeshiva University 1974 
20. Maradudin,A.A., Montroll,E.W., Weiss, G.H.: Theory of lattice dynamics in the harmonic approximation. New York: Academic Press 1963

21. Minlos, R. A. : Trudy. Moskov. Obsc. 8, 497 (1959) = Selected transl. in math. stat. and prob. 3, 291 (1963)

22. Kato,T.: Pertubation theory for linear operators. Berlin-Heidelberg-New York: Springer 1966

23. Ornstein,D.S. : Ergodic theory, randomness, and dynamical systems. New Haven: Yale University Press 1974

24. Pulvirenti,M.: Stability, equilibrium and KMS for infinite classical systems. Preprint 1976

25. Haag, R., Kastler,D., Trych-Pohlmeyer,E.: Commun. math. Phys. 38, 173 (1974)

26. Aizenman, M., Gallavotti,G., Goldstein, S., Lebowitz,J.L.: Commun. math. Phys. 48, 1 (1976)

27. Davies, E. B.: Commun math. Phys. 33, 171 (1973); J. Math. Phys. 15, 2036 (1974)

28. Pulè,J.: Commun. math. Phys. 38, 241 (1974)

29. Davies, E. B.: Commun. math. Phys. 39, 91 (1974)

30. Davies, E. B.: Math. Ann. 219, 147 (1976); Ann. Inst. H. Poinc. 11, 265 (1975)

31. Haake, F.: Statistical treatment of open systems by generalized master equations. Springer tracts in modern physics, Vol. 66. Berlin-Heidelberg-New York: Springer 1966

32. Narnhofer,H.: Acta Phys. Austr. 31, 349 (1970), 36, 217 (1972)

Hemmen,J.L.van, Vertogen, G.: Physica 81 A, 391 (1975)

33. See for example Jackson,E. A.: Nonlinearity and irreversibility in lattice dynamics. University of Illinois. Preprint (PL)-76-g

Communicated by J. Glimm

Received September 21, 1976 